\title{
Spin current and polarization reversal through a single-molecule magnet with ferromagnetic electrodes
}

\author{
Haiqing Xie, ${ }^{1}$ Qiang Wang, ${ }^{1}$ Bo Chang, ${ }^{2}$ Hujun Jiao, ${ }^{1}$ and J.-Q. Liang, ${ }^{1}$ 田 \\ ${ }^{1}$ Institute of Theoretical Physics and Department of Physics, Shanxi University, Taiyuan 030006, China \\ ${ }^{2}$ College of Physics and Optoelectronics, Taiyuan University of Technology, Taiyuan 030024, China
}

(Dated: August 7, 2018)

\begin{abstract}
We theoretically study the spin-polarized transport through a single-molecule magnet, which is weakly coupled to ferromagnetic leads, by means of the rate-equation approach. We consider both the ferromagnetic and antiferromagnetic exchange-couplings between the molecular magnet and transported electron-spin in the nonlinear tunneling regime. For the ferromagnetic exchangecoupling, spin current exhibits step- and basin-like behaviors in the parallel and antiparallel configurations respectively. An interesting observation is that the polarization reversal of spin-current can be realized and manipulated by the variation of bias voltage in the case of antiferromagnetic exchange-coupling with antiparallel lead-configuration, which may be useful in the development of spintronic devices, while the bias voltage can only affect the magnitude of spin-polarization in the ferromagnetic coupling.
\end{abstract}

PACS numbers: 75.50.Xx, 73.23.-b, 72.25.-b, 85.75.d

\section{INTRODUCTION}

In the past few years, electron transport through magnetic molecules, especially the single-molecule magnet (SMM), was intensively studied in both experimental 1 5] and theoretical[6-23] aspects, which is stimulated by the fundamental importance as well as potential applications in molecular spintronics. Many fascinating properties have been found, such as complex tunneling spectra [6, 7], negative differential conductance (NDC) [1, 8 -10], Kondo effect 12, 13], Berry phase blockade 14], full counting statistics [15], colossal spin fluctuations [16], and so on, which are resulted from the large spin-number and high anisotropy of SMM. The complete current suppression and negative differential conductance are also confirmed by the single-molecule-transistor measurement, which as a function of bias, gate voltage, and external magnetic field provide evidences of magnetic signatures of the SMM[1]. Manipulation of spins in magnetic molecules, which is based on the development of spin-controlling techniques, may result in new strategies of quantum-state control. It is demonstrated that the charge and spin states of molecule can be identified from the measured tunneling spectra due to the existence of exchange interaction between the local spin of magnetic molecule and the spin of tunneling electron, particularly the antiferromagnetic exchange interaction in a magnetic single-molecule transistor based on N@C60[4, 5]. On the other hand the tunneling current between magnetic electrodes can also control the orientation of molecular magnet shown by the study of colossal spin fluctuations [16].

Based on the study of spin polarized transport through a SMM some new spintronic devices are proposed, which display many interesting effects. In particular the spin-

*Electronic address: jqliang@sxu.edu.cn

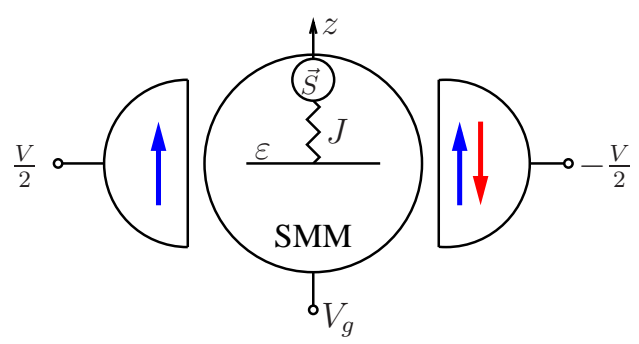

FIG. 1: (Color online) The schematic diagram of quantum transport through a SMM.

diode behavior [10] and tunnel magnetoresistance (TMR) [13, 17] are shown to be resulted from the exchange coupling between the lowest-unoccupied-malecular-orbital (LUMO) level and the core spin. Furthermore a large negative TMR is predicted in the case of antiferromagnetic exchange-coupling [17]. The giant spin amplification and spin-blockade behavior are useful in molecular spintronics to serve as a read-out mechanism [8, [9]. It is also found that the magnetization of SMM can be reversed by spin polarized current [8, 18 20] and spin-bias driven magnetization reversal is also observed theoretically 21]. The most recent study is focused on the manipulation of SMM by thermal spin-transfer torque 22]. Moreover a highly spin-polarized current can be generated by thermoelectric effects [23].

To date, most researches are concentrated on the manipulation of a SMM by the transported electron, which on the other hand may be used as a probe to explore the level structure of the SMM. The spin-polarized current through a SMM itself has received little attention, while in the quantum-dot (QD) case it is extensively studied 24 26] in terms of nonequilibrium Green's functions [25] and master-equation [26] respectively. The QD acts as a spin-current diode giving rise to the spin blockade. The spin current in QD system shows quite 
different characteristics compared with the charge transport [25, 27, 28].

We in the present paper study the spin-polarized transport through a SMM, which has more complex levelstructure than the single QD. Moreover the exchange coupling between SMM and the transported electron-spin leads to additional dynamic mechanism to manipulate the spin-polarization of current. Both parallel and antiparallel configurations of lead magnetization are considered along with the ferromagnetic and antiferromagnetic couplings. For the ferromagnetic exchange-coupling, the spin current variation with respect to the bias voltage exhibits a step-like curve in parallel configurations and basin-like behavior in the antiparallel configurations, respectively. The interesting observation in antiferromagnetic coupling case is that, the spin-polarization can be reversed with increasing bias voltage in antiparallel leadconfigurations.

\section{EXCHANGE COUPLING AND TRANSITION RATE OF TUNNELING}

We consider a system which consists of a SMM coupled to two ferromagnetic metallic-electrodes (see Fig. 1.), which can be described by the Hamiltonian [8, 9, 19]

$$
H=H_{S M M}+H_{\text {leads }}+H_{T} .
$$

The first term in Eq. (1) concerning the SMM of easyaxis anisotropy with parameter $K_{z}>0$ has the form

$$
H_{S M M}=\sum_{\sigma}\left(\varepsilon-e V_{g}\right) d_{\sigma}^{\dagger} d_{\sigma}+U d_{\uparrow}^{\dagger} d_{\uparrow} d_{\downarrow}^{\dagger} d_{\downarrow}-J \mathbf{s} \cdot \mathbf{S}-K_{z}\left(S^{z}\right)^{2},
$$

in which $J \mathbf{s} \cdot \mathbf{S}$ is the exchange interaction between electron spin and the giant spin $\mathbf{S}$ of SMM with $J$ being the exchange coupling parameter and $\mathbf{s} \equiv \sum_{\sigma \sigma^{\prime}} d_{\sigma}^{\dagger}\left(\sigma_{\sigma \sigma^{\prime}} / 2\right) d_{\sigma^{\prime}}$ is the corresponding spin operator of electron ( $\sigma$ is the vector of Pauli matrices). $d_{\sigma}^{\dagger}\left(d_{\sigma}\right)$ denotes the relevant electron creation (annihilation) operator and $\varepsilon$ is the single-electron energy of the LUMO level, which is tunable by the gate voltage $V_{g}$. U represents the Coulomb interaction of two electrons of opposite spins. The exchange interaction can be of either ferromagnetic $(J>0)$ or antiferromagnetic $(J<0)$ type.

The many-body states of electron-spin and molecule are expressed in terms of the eigenstates of operator $\mathbf{S}_{\text {tot }}^{z},\left|n, S_{t o t} ; m\right\rangle$, where $\mathbf{S}_{t o t}=\mathbf{s}+\mathbf{S}$ denotes the total spin operator of electron and molecule, with $n$ denoting the charge state of the SMM, $S_{t o t}$ the total spin quantumnumber and $m$ the eigenvalues of $\mathbf{S}_{\text {tot }}^{z}[8,[16,17,19]$. For the case of $n=0$, the state $\left|0, S_{\text {tot }}=S ; m\right\rangle \equiv$ $|0\rangle_{\text {orb }} \otimes|m\rangle_{\text {mol }}$ and the corresponding eigenenergy is $\varepsilon_{\left|0, S_{t o t}=S ; m\right\rangle}=-K_{z} m^{2}$, while when $n=2$, the state is $\left|2, S_{\text {tot }}=S ; m\right\rangle \equiv|\uparrow \downarrow\rangle_{\text {orb }} \otimes|m\rangle_{\text {mol }}$ and the eigenenergy is $\varepsilon_{\left|2, S_{t o t}=S ; m\right\rangle}=2\left(\varepsilon-e V_{g}\right)+U-K_{z} m^{2}$. For $n=1$ and $K_{z}-J / 2>0$, the state and eigenenergy are found as $\left|1, S_{\text {tot }}=S \pm 1 / 2 ; m\right\rangle \equiv a_{m \downarrow}^{( \pm)}|\downarrow\rangle_{\text {orb }} \otimes|m+1 / 2\rangle_{m o l}+$
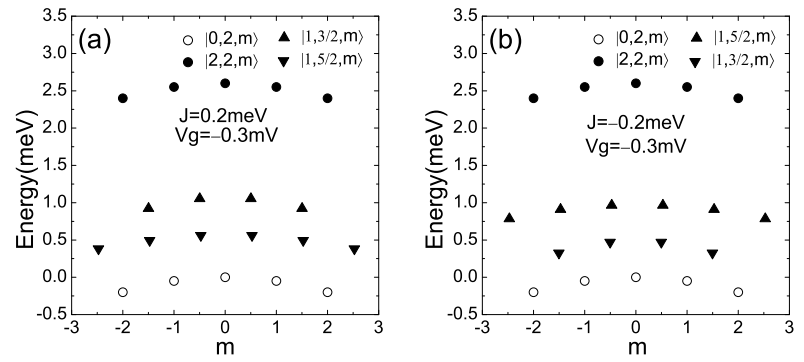

FIG. 2: (Color online) Energy spectra of the SMM for ferromagnetic (a) and antiferromagnetic (b) exchange- interactions as a function of magnetic quantum-number $m$ with $S=2$, $K_{z}=0.05 \mathrm{meV}, U=1 \mathrm{meV}, V g=-0.3 \mathrm{mV}$.

$b_{m \uparrow}^{( \pm)}|\uparrow\rangle_{o r b} \otimes|m-1 / 2\rangle_{m o l}$ and $\varepsilon_{\left|1, S_{\text {tot }}=S \pm 1 / 2 ; m\right\rangle}=(\varepsilon-$ $\left.e V_{g}\right)+J / 4-K_{z}\left(m^{2}+1 / 4\right) \pm \Delta E(m)$ respectively. For $n=1$ and $K_{z}-J / 2<0$, we have $\left|1, S_{\text {tot }}=S \mp 1 / 2 ; m\right\rangle \equiv$ $a_{m \downarrow}^{( \pm)}|\downarrow\rangle_{\text {orb }} \otimes|m+1 / 2\rangle_{m o l}+b_{m \uparrow}^{( \pm)}|\uparrow\rangle_{\text {orb }} \otimes|m-1 / 2\rangle_{m o l}$, and $\varepsilon_{\left|1, S_{t o t}=S \mp 1 / 2 ; m\right\rangle}=\left(\varepsilon-e V_{g}\right)+J / 4-K_{z}\left(m^{2}+\right.$ $1 / 4) \pm \Delta E(m)$, where $\Delta E(m)=\left[K_{z}\left(K_{z}-J\right) m^{2}+\right.$ $\left.(J / 4)^{2}(2 S+1)^{2}\right]^{1 / 2}$, and $a_{m \downarrow}^{( \pm)}, b_{m \uparrow}^{( \pm)}$are effective ClebschGordan coefficients [19].

The SMM is weakly coupled to two ferromagnetic metallic leads with the Hamiltonian given by

$$
H_{\text {leads }}=\sum_{\alpha=\mathbf{L}, \mathbf{R}} \sum_{\mathbf{k} \sigma} \varepsilon_{\alpha \mathbf{k}} c_{\alpha \mathbf{k} \sigma}^{\dagger} c_{\alpha \mathbf{k} \sigma},
$$

where $c_{\alpha \mathbf{k} \sigma}^{\dagger}\left(c_{\alpha \mathbf{k} \sigma}\right)$ is the creation (annihilation) operator for an electron of spin-index $\sigma$ and wave vector $\mathbf{k}$ in the $\alpha$ lead. The spin polarization of ferromagnetic lead $\alpha$ is defined as $P_{\alpha}=\left(\rho_{\alpha+}-\rho_{\alpha-}\right) /\left(\rho_{\alpha+}+\rho_{\alpha-}\right)$, with $\rho_{\alpha+(-)}$ denoting the density of states for the majority (minority) electrons in the $\alpha$ lead. In this paper, we assume that the magnetization of ferromagnetic leads is collinear with the easy axis of SMM. The tunneling processes between the molecule and leads can be described by Hamiltonian

$$
H_{T}=\sum_{\alpha \mathbf{k} \sigma}\left[t_{\alpha} c_{\alpha \mathbf{k} \sigma}^{\dagger} d_{\sigma}+t_{\alpha}^{*} d_{\sigma}^{\dagger} c_{\alpha \mathbf{k} \sigma}\right]
$$

with $t_{\alpha}$ denoting the tunnel matrix element between the molecule and $\alpha$ lead. The spin-dependent tunnel coupling-strength is given by

$$
\Gamma_{\alpha \sigma}=2 \pi \rho_{\alpha \sigma}\left|t_{\alpha}\right|^{2},
$$

and the total coupling-strength is $\Gamma_{\alpha}=\sum_{\sigma} \Gamma_{\alpha \sigma}$. In order to obtain transport properties in both the sequential and cotunneling regimes, we employ the rate-equation approach with the help of $T$-matrix 29 32, which satisfies the iterative equation

$$
T=H_{T}+H_{T} \frac{1}{E_{I}-H_{0}+i 0^{+}} T
$$

where $E_{I}$ is the energy of initial state $|I\rangle$, and $0^{+}$denotes a small quantity in the retarded Green function. The 
transition strength from initial state $|I\rangle$ to final state $|F\rangle$ can be evaluated by the perturbation expansion of $T$ matrix in terms of the generalized Fermi's golden rule [29],

$$
\begin{aligned}
\Gamma_{F I} & =\frac{2 \pi}{\hbar}|\langle F|T| I\rangle|^{2} \delta\left(E_{F}-E_{I}\right) \\
& =\frac{2 \pi}{\hbar}\left|\left\langle F\left|H_{T}+H_{T} \frac{1}{E_{I}-H_{0}+i 0^{+}} H_{T}+\ldots\right| I\right\rangle\right|^{2} \\
& \delta\left(E_{F}-E_{I}\right),
\end{aligned}
$$

where $|I\rangle$ and $|F\rangle$ are actually product states of the electron-lead and molecule. After eliminating the lead degree of freedom, the sequential transition rate up to the second-order of tunneling Hamiltonian $H_{T}$ can be obtained from the transition-strength formula

$$
\begin{aligned}
W_{\alpha \sigma}^{i, i^{\prime}} & =\frac{\Gamma_{\alpha \sigma}}{\hbar}\left[f_{\alpha}\left(\varepsilon_{i^{\prime}}-\varepsilon_{i}-\mu_{\alpha}\right)\left|\left\langle i^{\prime}\left|d_{\sigma}^{\dagger}\right| i\right\rangle\right|^{2}\right. \\
& \left.+\left[1-f\left(\varepsilon_{i}-\varepsilon_{i^{\prime}}-\mu_{\alpha}\right)\right]\left|\left\langle i^{\prime}\left|d_{\sigma}\right| i\right\rangle\right|^{2}\right],
\end{aligned}
$$

which describes the transition of molecule from state $|i\rangle$ to $\left|i^{\prime}\right\rangle$, due to spin- $\sigma$ electron tunneling into or out of lead $\alpha$. Where $\mu_{L}=-e V / 2, \mu_{R}=e V / 2, \varepsilon_{i}$ is the energy of state $|i\rangle$ and $f(x)$ is the Fermi distribution function. For the SMM model we can find the sequential transition selection-rule : $|\Delta n|=1,\left|\Delta S_{\text {tot }}\right|=1 / 2$, and $|\Delta m|=1 / 2$. The fourth-order cotunneling transition rate

$$
\begin{aligned}
& W_{\alpha \sigma, \alpha^{\prime} \sigma^{\prime}}^{i, i^{\prime}} \\
& =\frac{\Gamma_{\alpha \sigma} \Gamma_{\alpha^{\prime} \sigma^{\prime}}}{2 \pi \hbar} \int d \varepsilon f\left(\varepsilon-\mu_{\alpha}\right)\left[1-f\left(\varepsilon-\varepsilon_{i^{\prime}}+\varepsilon_{i}-\mu_{\alpha^{\prime}}\right)\right] \\
& \left|\sum_{j} \frac{\left\langle i^{\prime}\left|d_{\sigma}^{\dagger}\right| j\right\rangle\left\langle j\left|d_{\sigma^{\prime}}\right| i\right\rangle}{\varepsilon-\varepsilon_{i^{\prime}}+\varepsilon_{j}-i 0^{+}}+\frac{\left\langle i^{\prime}\left|d_{\sigma^{\prime}}\right| j\right\rangle\left\langle j\left|d_{\sigma}^{\dagger}\right| i\right\rangle}{\varepsilon+\varepsilon_{i}-\varepsilon_{j}+i 0^{+}}\right|^{2}
\end{aligned}
$$

stands for the virtual transitions from molecular states $|i\rangle$ to $\left|i^{\prime}\right\rangle$ while changing a spin- $\sigma$ electron of lead $\alpha$ into spin- $\sigma^{\prime}$ electron of lead $\alpha^{\prime}$. The divergence of above expressions existed whenever energy denominators vanish has to be eliminated by some regularization procedures [31 36] and in this paper we adopt the method described in Ref .[36]. The cotunneling transition selection rules are seen to be $|\Delta n|=0$, and $|\Delta m|=0, \pm 1$.

For the weak coupling between lead and molecule, i.e. $\Gamma_{\alpha \sigma} \ll k_{B} T$, electron transport can be considered as a stochastic Markovian process and thus the time evolution is described by the following rate equations

$$
\begin{aligned}
\frac{d P_{i}}{d t} & =\sum_{\alpha \alpha^{\prime} \sigma \sigma^{\prime} i^{\prime} \neq i}-\left(W_{\alpha \sigma, \alpha^{\prime} \sigma^{\prime}}^{i, i^{\prime}}+W_{\alpha \sigma}^{i, i^{\prime}}\right) P_{i} \\
& +\sum_{\alpha \alpha^{\prime} \sigma \sigma^{\prime} i^{\prime} \neq i}\left(W_{\alpha \sigma}^{i^{\prime}, i}+W_{\alpha^{\prime} \sigma^{\prime}, \alpha \sigma}^{i^{\prime}, i}\right) P_{i^{\prime}},
\end{aligned}
$$

where $P_{i}$ is the probability of finding molecule in the many-body state $i$. The stationary probabilities obtained

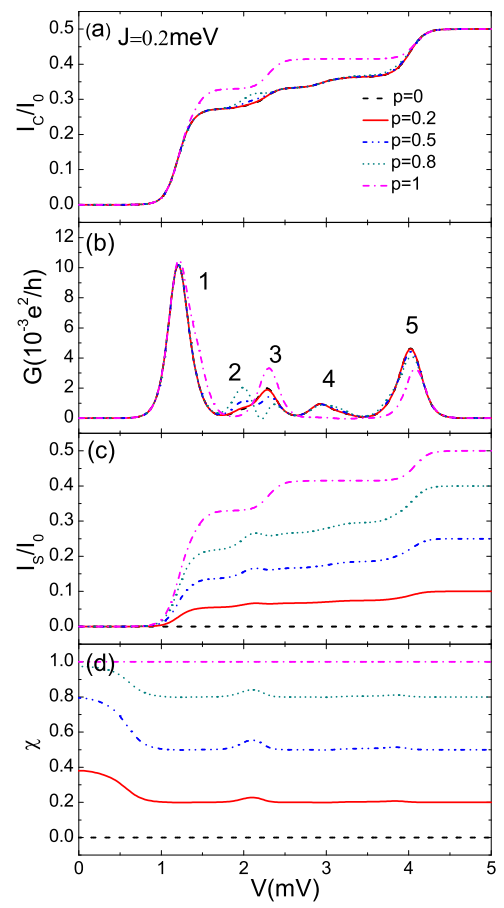

FIG. 3: (Color online) (a) Charge current $I_{c}$, (b) differential conductance $G$, (c) spin current $I_{s}$, and (d) current polarization $\chi$ as a function of the bias voltage $V$ for different lead-polarizations $p$ and ferromagnetic exchange coupling $(J=0.2 \mathrm{meV})$ in case of parallel configuration of leadmagnetization.

from the condition, $\frac{d P_{i}}{d t}=0$, with the transition rate together result in the current of spin- $\sigma$ through lead $\alpha$ :

$$
\begin{aligned}
I_{\alpha \sigma} & =-e(-1)^{\delta_{R \alpha}} \sum_{\alpha^{\prime} \neq \alpha \sigma^{\prime} i i^{\prime}}\left[\left(n_{i^{\prime}}-n_{i}\right) W_{\alpha \sigma}^{i, i^{\prime}} P_{i}\right. \\
& \left.+\left(W_{\alpha \sigma, \alpha^{\prime} \sigma^{\prime}}^{i, i^{\prime}}-W_{\alpha^{\prime} \sigma^{\prime}, \alpha \sigma}^{i, i^{\prime}}\right) P_{i}\right],
\end{aligned}
$$

from which we obtain the charge current $I_{\alpha}=I_{\alpha \uparrow}+$ $I_{\alpha \downarrow}$ and the spin current $I_{\alpha s}=I_{\alpha \uparrow}-I_{\alpha \downarrow}$. The current polarization is defined by $\chi=\left(I_{\alpha \uparrow}-I_{\alpha \downarrow}\right) /\left(I_{\alpha \uparrow}+I_{\alpha \downarrow}\right)$.

\section{CURRENTS AND POLARIZATIONS IN THE NONLINEAR REGIME}

The numerical results of the charge current, differential conductance, spin current, and current polarization in the nonlinear regime are carried out with parameters chosen as $S=2, \varepsilon_{d}=0.5 \mathrm{meV}, V g=-0.3 \mathrm{mV}, J=0.2 \mathrm{meV}$, $U=1 \mathrm{meV}, K_{z}=0.05 \mathrm{meV}$ and $k_{B} T=0.04 \mathrm{meV}$. We set the tunnel coupling between SMM and ferromagnetic leads to be $\Gamma=\Gamma_{L}=\Gamma_{R}=0.001 \mathrm{meV}$ and assume the same polarizations of two leads i.e., $p_{L}=p_{R}=p$. In addition, the current and differential conductance are scaled in units of $I_{0}=2 e \Gamma / \hbar$ and $G_{0}=10^{-3} e^{2} / h$, respectively. With the chosen parameters the numerical energy-spectrum of SMM is obtained as in Fig.2, from 


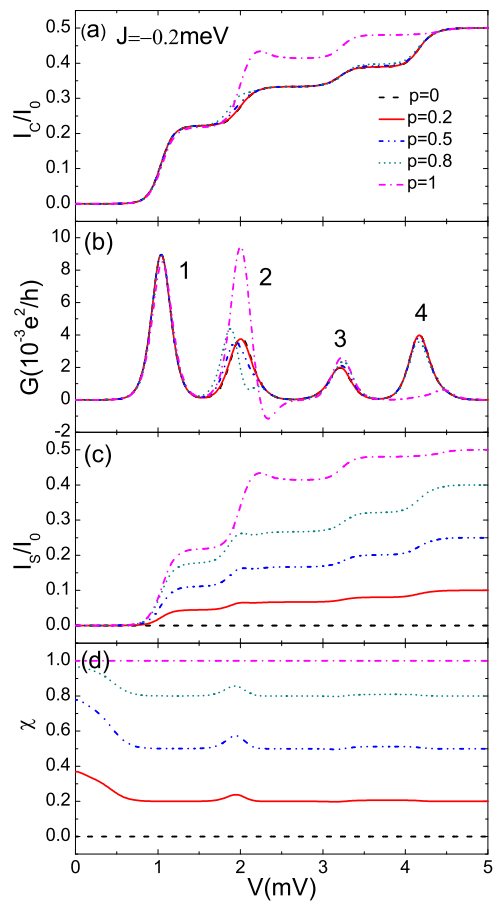

FIG. 4: (Color online) (a) Charge current $I_{c}$, (b) differential conductance $G$, (c) spin current $I_{s}$, and (d) current polarization $\chi$ as a function of the bias voltage $V$ for different lead-polarizations $p$ and antiferromagnetic exchange coupling $(J=-0.2 \mathrm{meV})$ in case of parallel configuration of leadmagnetization.

which one can find that the energy-eigenvalue of SMMstates corresponding to the total spin-number $S_{t o t}=5 / 2$ is lower than that of $S_{t o t}=3 / 2$ for the ferromagnetic exchange-interaction (Fig. 2(a)), while the situation is just opposite for the antiferromagnetic case (Fig. 2(b)). In what follows the spin currents are analyzed for two types of exchange interactions with ferromagnetic leads of both parallel and antiparallel configurations respectively.

\section{A. Parallel configuration}

For the ferromagnetic exchange-interaction, the biasvoltage dependences of charge current $I_{c}$ and differential conductance $G$ for different lead-polarizations $p$ are shown in Fig. 3(a) and Fig. 3(b) respectively, where it is seen that the deviations of $I_{c}$ and $G$ are negligibly small when the lead polarization varies in the region $p<1$. This observation is, as a matter of fact, the same as in the QD [27], where the resonant probability is independent of the lead-polarization $p$ [37]. There exist five evident resonant-peaks in Fig. 3(b), in which the first (corresponding to the transition $|0,2 ; \pm 2\rangle \Leftrightarrow$ $|1,5 / 2 ; \pm 5 / 2\rangle)$ and fifth $(|1,5 / 2 ; \pm 5 / 2\rangle \Leftrightarrow|2,2 ; \pm 2\rangle)$ peaks are higher than the other three resulted from the transitions: $|0,2 ; \pm 1\rangle \Leftrightarrow|1,3 / 2 ; \pm 3 / 2\rangle(2),|0,2 ; \pm 2\rangle \Leftrightarrow$
$|1,3 / 2 ; \pm 3 / 2\rangle(3)$ and $|1,3 / 2 ; \pm 3 / 2\rangle \Leftrightarrow|2,2 ; \pm 2\rangle$ (4), respectively [17]. However, for the full polarization $p=1$, the current and differential conductance are quite different since only one spin-up channel is involved in the transition without competition between majority and minority spin channels, which leads to dynamic spinblockade [9, 38]. The bias-voltage dependence of the spin current $I_{s}$ and current-polarization $\chi$ for various leadpolarizations $p$ are shown in Fig. 3(c) and Fig. 3(d). The spin current exhibits step-like behavior in parallel lead-configurations, which is also the same as in a single QD [27], while with more steps due to the complex energy levels of SMM. In addition, when lead-polarization $p$ increases, the spin current varies more evidently along with an increasing magnitude of current polarization $\chi$ in contrast to the charge current. The current polarization $\chi$ approaches the largest value at a very low bias voltage, which is dominated by elastic co-tunneling processes through majority-majority and minority-minority spin channels. With increasing bias voltage, the inelastic co-tunneling starts to enter the transport and leads to the decrease of $\chi$. Moreover, as the bias voltage reaches the threshold of sequential transport, the value of $\chi$ approaches the lead-polarization $p$ [26], due to the appearance of sequential transport channels.

The results of antiferromagnetic exchange interaction (Fig.4) are different from the ferromagnetic case due to the two degenerate ground-states $|1,3 / 2 ; \pm 3 / 2\rangle$ seen from Fig. 2(b), where the differential conductance spectra have four evident peaks. With the increase of lead-polarization $p$ (except $p=1$ ) the position of peak-2 shifts toward the negative direction of the $V$-axis since the contribution is mainly from the transition of $|0,2 ; \pm 1\rangle \Leftrightarrow|1,5 / 2 ; \pm 3 / 2\rangle$ instead of $|0,2 ; \pm 2\rangle \Leftrightarrow|1,5 / 2 ; \pm 5 / 2\rangle$. The other three peaks (peak $-1,-3,-4)$ are resulted from the transitions of $|0,2 ; \pm 2\rangle \Leftrightarrow|1,3 / 2 ; \pm 3 / 2\rangle,|1,5 / 2 ; \pm 5 / 2\rangle \Leftrightarrow|2,2 ; \pm 2\rangle$, and $|1,3 / 2 ; \pm 3 / 2\rangle \Leftrightarrow|2,2 ; \pm 2\rangle$, respectively. For the case of $p=1$, the position of peak-2 has a great change different from the peak-1 in the ferromagnetic coupling, where the state $|0,2 ; 2\rangle$ does not participate in transition. Besides, $I_{s}$ and $\chi$ exhibit similar behaviors with the ferromagnetic case.

\section{B. Antiparallel configuration and current polarization reversal}

In the antiparallel configuration of magnetic leads peculiar behavior of the spin current is observed, which is shown in Fig. 5. From Fig. 5(a), it is seen that $I_{c}$ decreases monotonously from a maximum value to zero at a certain bias-voltage when $p$ increases from 0 to 1 . Since transport occurs mainly through two spin channels (majority-minority and minority-majority), for a large $p$ the most probable transport process is that a spin-down electron of higher rate from the lead $R$ tunnels through the molecule along with a spin-flip and molecular spin- 


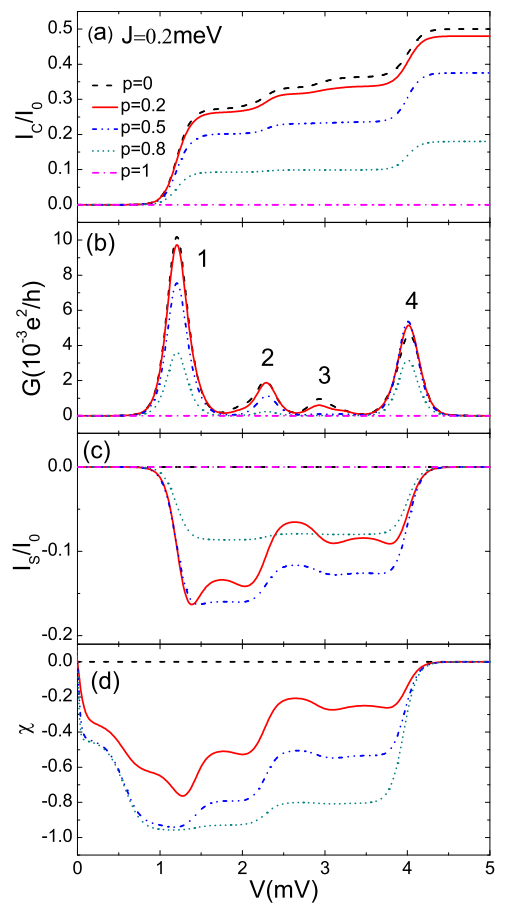

FIG. 5: (Color online) (a) Charge current $I_{c}$, (b) differential conductance $G$, (c) spin current $I_{s}$, and (d) current polarization $\chi$ as a function of the bias voltage $V$ for different lead-polarizations $p$ with ferromagnetic exchange coupling $(J=0.2 \mathrm{meV})$ and antiparallel lead-configuration.

state lowering simultaneously. However, this process is limited by the lowest spin-state of molecule and thus the charge current finally vanishes [9]. Also, the $G$ peaks in Fig. 5(b) are suppressed with the increase of $p$ and the peak-2 appeared in Fig. 3(b) even disappears because the transition channels through states $|0,2 ; \pm 1\rangle$ are blocked.

The spin current $I_{s}$ (see Fig. $5(\mathrm{c})$ ) shows a basinlike behavior in the region $I_{\uparrow}<I_{\downarrow}$ similar to the QD case. The difference is that $I_{s}$ in the SMM can be suppressed by the lead-polarization $p$ at some value, however it is enhanced always with the increase of $p(p \neq 1)$ in the QD. Nevertheless, $\chi$ displays a monotonic behavior with increase of $p(p \neq 1)$ for a fixed bias voltage $V$ seen from Fig. 5(d). It is found that the polarization $\chi$ can have a high value at low lead-polarization $p$ resulted from the exchange coupling between the electronspin and the SMM. Moreover, in contrast to the parallel lead-configuration the current with $\chi=0$ is contributed mainly from elastic co-tunneling processes around zero bias voltage, which belong to the majority-minority and minority-majority spin channels. With increasing $V$, the inelastic co-tunneling dominates the transport and $\chi$ decreases. Beyond the threshold value of sequential transport the peak- 1 arises and $\chi$ reaches the lowest value due to the transition $|0,2 ;-2\rangle \Leftrightarrow|1,5 / 2 ;-5 / 2\rangle$. Then $\chi$ increases since more transport channels enter the bias window.

Fig. 6 is the $I_{c}-V$ diagram for antiferromagnetic

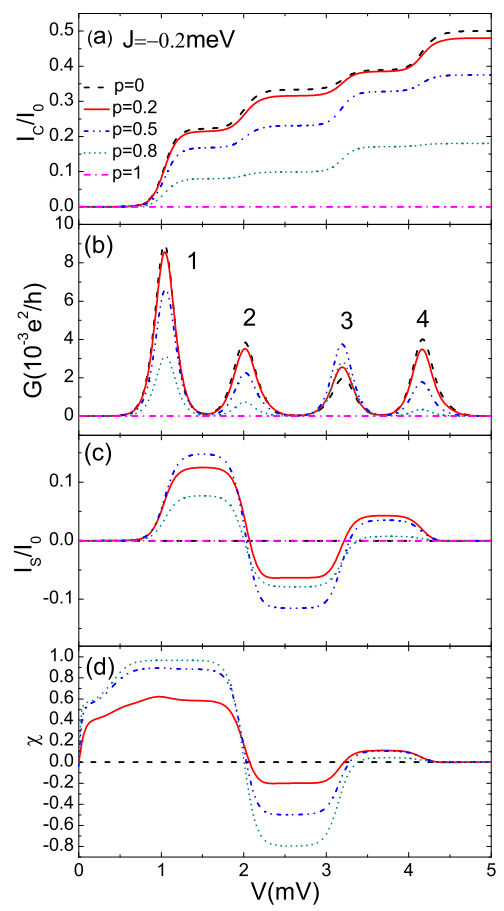

FIG. 6: ((Color online) (a) Charge current $I_{c}$, (b) differential conductance $G,(\mathrm{c})$ spin current $I_{s}$, and (d) current polarization $\chi$ as a function of the bias voltage $V$ for different lead-polarizations $p$ with antiferromagnetic exchange coupling $(J=-0.2 \mathrm{meV})$ and antiparallel lead-configuration.

exchange coupling, in which the $I_{c}$ curve (Fig. 6(a)) exhibits a similar behavior as in the ferromagnetic coupling case. The corresponding differential conductance $G$ is shown in Fig. 6(b). Moreover, in the antiparallel lead-configuration the steady state probabilities with the negative eigenvalues of spin operator $S_{t o t}^{z}$ become larger than that of positive eigenvalues resulted by the spin-flip process 9 , 16, 17]. Therefore, the peak-1 and peak-3 of the $G$ curve are mainly contributed by the spin-up electron transitions $|0,2 ;-2\rangle \Leftrightarrow|1,3 / 2 ;-3 / 2\rangle$ and $|1,5 / 2 ;-5 / 2\rangle \Leftrightarrow|2,2 ;-2\rangle$ respectively. On the other hand, the peak-2 (peak-4) is mainly induced by the transition $|0,2 ;-2\rangle \Leftrightarrow|1,5 / 2 ;-5 / 2\rangle(|1,3 / 2 ;-3 / 2\rangle \Leftrightarrow$ $|2,2 ;-2\rangle)$ of the spin-down electron. The spin-current curve $I_{s}$ in Fig. 6(c) has a very interesting characteristic different from the ferromagnetic case that it possesses an alternate structure of two-plateau and one-basin. The first plateau appears between the positions of peak-1 and peak-2, where the spin-up current is larger than the spin-down (positive $\chi$ ). In addition, the spin current shows a non-monotonic behavior with increasing leadpolarizations $p$, which is resulted from the competition between the spin-up and spin-down currents. Furthermore, with increasing the bias voltage the basin emerges when the transition $|0,2 ;-2\rangle \Leftrightarrow|1,5 / 2 ;-5 / 2\rangle$ starts to participate in the transport, in which the spin-down current becomes larger than the spin-up current (negative $\chi)$. The second plateau of spin current appears as the 
bias voltage increases to the value between the peak-3 and peak-4. Finally when all transport channels open up, the spin current vanishes. In conclusion, the polarization of spin current $\chi$ can be inverted by adjusting the bias voltage and the spin current exhibits rich multi-NDC behaviors [39]. The polarization reversal of spin current may have potential application in the spintronic device.

\section{CONCLUSION}

In summary, the spin current through a SMM weakly coupled to two ferromagnetic leads is obtained in the nonlinear tunneling regime for both ferromagnetic and antiferromagnetic exchange-couplings. The complex en- ergy spectrum of the SMM results in rich properties of the currents and conductances compared with the single $\mathrm{QD}$, which on the other hand provide magnetic signatures of the SMM. The most interesting observation is the spin-polarization reversal in the antiferromagnetic exchange-coupling case, which can be manipulated by the bias voltage. These theoretical results may be useful in the future development of spintronic devices.

\section{ACKNOWLEDGMENT}

This work was supported by National Natural Science Foundation of China (Grant No. 11075099, No. 11004124 and No. 10974124).
[1] H. B. Heersche et al., Phys. Rev. Lett. 96, 206801 (2006).

[2] M.-H. Jo et al., Nano Lett. 6, 2014 (2006).

[3] A. S. Zyazin et al., Nano Lett. 10, 3307 (2010).

[4] J. E. Grose et al., Nat. Mater. 7, 884 (2008).

[5] N. Roch et al., Phys. Rev. B 83, 081407(R) (2011).

[6] G.-H. Kim and T.-S. Kim, Phys. Rev. Lett. 92, 137203 (2004); C. Romeike, M. R. Wegewijs, and H. Schoeller, Phys. Rev. Lett. 96, 196805 (2006); J. Fernández-Rossier and R. Aguado, Phys. Rev. Lett. 98, 106805 (2007); J. Lehmann and D. Loss, Phys. Rev. Lett. 98, 117203 (2007); C. Romeike et al., Phys. Rev. B 75, 064404 (2007).

[7] F. Elste and C. Timm, Phys. Rev. B 71, 155403 (2005).

[8] C. Timm and F. Elste, Phys. Rev. B 73, 235304 (2006).

[9] F. Elste and C. Timm, Phys. Rev. B 73, 235305 (2006).

[10] M. Misiorny and J. Barnaś, Europhys. Lett. 89, 18003 (2010).

[11] F. Elste and C. Timm, Phys. Rev. B 75, 195341 (2007).

[12] C. Romeike et al., Phys. Rev. Lett. 96, 196601 (2006);

C. Romeike et al., Phys. Rev. Lett. 97, 206601 (2006); M. N. Leuenberger and E. R. Mucciolo, Phys. Rev. Lett. 97, 126601 (2006); G. Gonzalez, M. N. Leuenberger, and E. R. Mucciolo, Phys. Rev. B 78, 054445 (2008); R.-Q. Wang and D. Y. Xing, Phys. Rev. B 79, 193406 (2009); F. Elste and C. Timm, Phys. Rev. B 81, 024421 (2010).

[13] M. Misiorny, I. Weymann, and J. Barnaś, Phys. Rev. B 84, 035445 (2011); M. Misiorny, I. Weymann, and J. Barnaś, Phys. Rev. Lett. 106, 126602 (2011).

[14] G. González and M. N. Leuenberger, Phys. Rev. Lett. 98, 256804 (2007).

[15] K.-I. Imura, Y. Utsumi, and T. Martin, Phys. Rev. B 75, 205341 (2007); H.-B. Xue, Y.-H. Nie, Z.-J. Li, and J.Q. Liang, J. Appl. Phys. 108, 033707 (2010); H.-B. Xue, Y.-H. Nie, Z.-J. Li, and J.-Q. Liang, J. Appl. Phys. 109, 083706 (2011); H.-B. Xue, Y.-H. Nie, Z.-J. Li, and J.-Q. Liang, Phys. Lett. A 375, 716 (2011).

[16] T. Jonckheere, K.-I. Imura, and T. Martin, Phys. Rev. B 78, 045316 (2008).

[17] M. Misiorny and J. Barnaś, Phys. Rev. B 79, 224420 (2009).

[18] M. Misiorny and J. Barnaś, Phys. Rev. B 75, 134425 (2007).

[19] M. Misiorny and J. Barnaś, Phys. Rev. B 76, 054448 (2007).
[20] M. Misiorny and J. Barnaś, Phys. Rev. B 77, 172414 (2008).

[21] H.-Z. Lu, B. Zhou, and S.-Q. Shen, Phys. Rev. B 79, 174419 (2009).

[22] Z. Zhang, L. Jiang, R. Wang, B. Wang, and D. Y. Xing, Appl. Phys. Lett. 99, 133110 (2011).

[23] R.-Q. Wang et al., Phys. Rev. Lett. 105, 057202 (2010); Z. Zhang et al., Appl. Phys. Lett. 97, 242101 (2010).

[24] J. König and J. Martinek, Phys. Rev. Lett. 90, 166602 (2003); M. Braun, J. König and J. Martinek, Phys. Rev. B 70, 195345 (2004).

[25] F. M. Souza, A. P. Jauho, and J. C. Egues, Phys. Rev. B 78, 155303 (2008).

[26] F. M. Souza, J. C. Egues, and A. P. Jauho, Phys. Rev. B 75, 165303 (2007).

[27] H.-F. Mu, G. Su, and Q.-R. Zheng, Phys. Rev. B 73, 054414 (2006).

[28] R. Y. Yuan, R. Z. Wang, and H. Yan, J. Phys. Condens. Matter 19, 376215 (2007).

[29] H. Bruus and K. Flensberg, Many-body Quantum Theory in Condensed Matter Physics (Oxford University Press, Oxford, 2004).

[30] C. Timm, Phys. Rev. B 77, 195416 (2008).

[31] J. Koch, F. von Oppen, Y. Oreg, and E. Sela, Phys. Rev. B 70, 195107 (2004).

[32] J. Koch, F. von Oppen, and A. V. Andreev, Phys. Rev. B 74, 205438 (2006).

[33] M. Turek and K. A. Matveev, Phys. Rev. B 65, 115332 (2002).

[34] S. Koller, M. Grifoni, M. Leijnse, and M. R. Wegewijs, Phys. Rev. B 82, 235307 (2010).

[35] C. Timm, Phys. Rev. B 83, 115416 (2011).

[36] I. Weymann and J. Barnaś, Eur. Phys. J. B 46, 289 (2005).

[37] N. Sergueev, Q. F. Sun, H. Guo, B. G. Wang, and J. Wang, Phys. Rev. B 65, 165303 (2002).

[38] A. Cottet and W. Belzig, Europhys. Lett. 66, 405 (2004); A. Cottet, W. Belzig, and C. Bruder, Phys. Rev. Lett. 92, 206801 (2004); A. Cottet, W. Belzig, and C. Bruder, Phys. Rev. B 70, 115315 (2004).

[39] H.-H. Fu and K.-L. Yao, J. Appl. Phys. 110, 094502 (2011). 